\title{
MISZELLEN
}

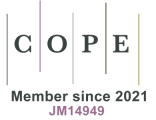

https://doi.org/10.18778/2196-8403.2021.11

HENK DE BERG

\section{Der engagierte Beobachter. Über TZVETAN TODOROV ${ }^{1}$}

TZVETAN TODOROV starb am 7. Februar 2017. Was hat er uns hinterlassen? Was bleibt? Die These der nachfolgenden Überlegungen lautet: Mehr noch als seine spezifischen Beiträge zur Literatur- und Kulturtheorie sowie zur Geschichte der Philosophie ist TODOROVs intellektueller Ansatz sein eigentliches Erbe.

TODOROV war ein europäischer Intellektueller; ich würde sogar sagen, er war der europäische Intellektuelle schlechthin. Er sprach mehrere Sprachen fließend, besaß eine außergewöhnliche Allgemeinbildung und war genuin weltoffen. Das Nachrichtenmagzin Der Spiegel drückte es gut aus, als es TODOROVs Nachruf wie folgt einleitete: „Wenn man einem Außerirdischen erklären wollte, was ein europäischer Intellektueller ist, müsste man ihm von TZVETAN TODOROV erzählen“ (Der Spiegel:123). Dieser Nachruf zeigt nolens volens freilich auch etwas anderes, die Tatsache nämlich, dass Todorov nie den Status eines intellektuellen Celebritys erreichte: Die Würdigung im Spiegel beschränkt sich auf eine einzige Spalte. TODOROV war ein Denker höchsten Ranges. Er wurde aber viel weniger gelesen und war der breiten Öffentlichkeit viel weniger bekannt als viele Stars der Intelligenz, deren Publikationen in Bahnhofs- und Flughafenbuchhandlungen zu finden sind. Es gibt dafür eine auf den ersten Blick befremdlich anmutende Erklärung: TODOROV war in politisch-philosophischer

1 Revidierte und leicht erweiterte Fassung eines Vortrags auf dem internationalen Gedenkkolloquium Hommage à Tzvetan Todorov in Paris am 11. Juli 2017. Autorisierte Übersetzung aus dem Französischen von David Engels. 
Hinsicht ein Vertreter der Mitte, ein Verteidiger des westeuropäischen Nachkriegsmodells eines Rechts- und Sozialstaates.

Wir haben es hier mit einem höchst paradoxen Umstand zu tun: Das Selbstbewusstsein des modernen Menschen konstituiert sich in und durch die revolutionären politischen und intellektuellen Umwälzungen des 18. Jahrhunderts, das heißt als radikale Kritik am Bestehenden, und wenn dieser misstrauische Blick einmal etabliert ist, so lässt er jede Handlung und jede Institution als verdächtig erscheinen, dergestalt, dass sich noch die bewundernswertesten sozialen Errungenschaften als unzureichend ausnehmen - als Übel, die es durch makellose Alternativen zu ersetzen gilt. In der Formulierung des Politologen Bernard Willms:

Die Geschichte der bürgerlichen Gesellschaft ist die Geschichte des Kampfes des Bürgers gegen den Bürger. Gegenüber dem ,Philister', also dem konkreten Bürger in seiner institutionell-positiven Beschränktheit, behauptete sich immer auch der sich auf das Allgemeine, auf den Menschen beziehende Bürger: als Genie oder Bohemien, als Weltverbesserer oder Revolutionär oder als kritischer oder protestierender Intellektueller. [...] Die bürgerliche Gesellschaft hatte sich revolutionär etabliert; das Selbstverständnis, mit dem die feudale Privilegiengesellschaft revolutionär überwunden wurde, war das Selbstbewußtsein des Bürgers als Allgemeines: Der Bürger machte seine Revolution als Mensch. [...] Aber damit stellte sich die bürgerliche Gesellschaft unter das Gesetz dieses Anfangs: der Bürger als allgemeiner, als Mensch also, blieb immer die Negation des Bürgers und seiner Gesellschaftsform als besonderer. (WILLMS 1969:9) ${ }^{2}$

Das heißt konkret: Allzu oft erscheinen nun sogar die Garantien der Freiheit als gesellschaftliche Fesseln, die Institutionen des Rechtsstaates als Instrumente der Repression. Und die selbsternannten Vertreter dieses hyperkritischen Bewusstseins sorgen dafür, dass nichts dieser Herrschaft des Verdachts entkommt. $^{3}$

TZVETAN TODOROV indessen stand nie auf der Seite solcher politisch-philosophischen Wachhunde (von rechts oder links), die heute ständig über den Bildschirm flimmern und es sich zum Ziel gesetzt haben, den intellektuellen Diskurs

2 In Hegelscher Formulierung: Die kontinuierliche Negation der Negation - die Dialektik von Problemlösungen und Lösungsproblematisierungen - ist das Grundprinzip der Moderne.

[Anm. d. Übers.:] Zum Begriff ,Herrschaft des Verdachts' siehe den Abschnitt „Die absolute Freiheit und der Schrecken“ in Hegels Phänomenologie des Geistes (HEGEL 1986:431-441). 
zu überwachen und diejenigen zu bestrafen, die vom rechten Weg abweichen. ${ }^{4}$ Er gehörte ebenfalls nicht zu jenen - von den Medien genauso begeistert umarmten - Alarmisten, die in regelmäßigen Abständen die Niederlage des Denkens, die Selbstabschaffung Frankreichs oder gar das Ende der Welt verkünden (vgl. FINKIELKRAUT 1989; ZEMMOUR 2014; ŽIŽEK 2011). Und er gehörte schon gar nicht zu jenen Weltverbesserern, die genau wissen, wie sich eine solche Katastrophe vermeiden ließe - wenn man nur ihrem Rat folgen würde.

Als KARINE ZBINDEN und ich TODOROV im März 2015 interviewten, war es das erste Mal, dass wir ausführlicher miteinander sprachen. Drei Dinge sind mir besonders stark in Erinnerung geblieben. Da war zunächst das völlige Fehlen von Eitelkeit:

Wissen Sie [so sagte er uns], ich nehme nicht in Anspruch, ein Philosoph zu sein. Die Philosophie ist nicht mein eigentliches Arbeitsgebiet. Ich betrachte mich eher als Historiker - ein Ideenhistoriker, der natürlich bestimmte theoretische Ausgangspunkte besitzt und bestimmte Positionen vertritt -, aber nicht als Denker, der sozusagen auf Augenhöhe mit den großen Philosophen der Vergangenheit diskutieren würde. (DE BERG / ZBINDEN 2017:12)

Das zweite, was mir imponierte, war seine unglaubliche intellektuelle Beweglichkeit, seine Geistesgegenwart. Ich erinnere mich, dass ich drei ebenso komplexe wie detaillierte Fragen zu Sudhir Hazareesinghs gerade erschienenem Buch How the French Think stellte (vgl. HAZAREESINGH 2015). TODOROV gab eine lange Antwort auf die erste Frage, eine ähnlich ausführliche Antwort auf die zweite Frage, und ging dann zum dritten Punkt über - den ich selbst bereits vergessen hatte!

Nicht zuletzt beeindruckten mich seine besonnene Redeweise und seine intellektuelle Fairness. Er hasste jede Art von Pathos und versuchte immer, beide Seiten der Medaille zu sehen. Was auch immer das Thema unserer Fragen war - sei es der hasserfüllte Nationalismus der extremen Rechten, die zunehmende Mediatisierung der Gesellschaft oder die potentielle ,transhumanistische' Robotisierung des Menschen -, TODOROVs Antworten waren ausnahmslos höchst reflektiert und verantwortungsbewusst. Am meisten hat mir imponiert: die dezidierte Weigerung, den verschiedenen heute so überaus medienwirksamen politisch-philosophischen Alarmismen - allen voran dem kulturpessimisti-

4 [Anm. d. Übers.:] Der Begriff des politisch-philosophischen ,Wachhundes“ stammt aus NizAN 1969; vgl. auch HALIMI 1997 (erweiterte und revidierte Fassung 2005), woraus 2012 ein viel beachteter Dokumentarfilm wurde. Die Verben ,überwachen' und ,bestrafen“ spielen auf Michel Foucaults Surveiller et punir an (FOUCAULT 1977). 
schen déclinisme und dessen spiegelbildlicher Verkehrung, der utopischen Exaltation - das Wort zu reden, will heißen der Wille, sich die Urteilsfähigkeit zu bewahren. Dieses denkerische Prinzip (denn das war es: ein Prinzip, ein philosophisches Credo, und nicht bloß eine psychologische Disposition) hinderte ihn nicht daran, konsequent und eindeutig Position zu beziehen. Außerdem hatte dieses Prinzip nichts mit einem naiven Szientismus zu tun. Für TODOROV bestand die Aufgabe des Denkens darin, die Dinge so zu sehen, wie sie sind, und sie als solche zu verstehen; und zwar gerade deshalb, weil wir immer in das Objekt, das wir analysieren, involviert sind, involviert mit unseren theoretischen Vorannahmen und persönlichen Überzeugungen. Es ging ihm mit anderen Worten immer um ein Doppeltes: darum, dass der erforschte Gegenstand zu uns spricht (geisteswissenschaftliche Objektivität betrachtete er als eine Illusion), aber ebenso darum, dass es das Objekt ist, das zu uns spricht (den postmodernen Relativismus und den radikalen Konstruktivismus hielt er für genauso verfehlt). Wie RAYMOND ARON war auch TZVETAN TODOROV ein engagierter Beobachter (ARON 1983).

Während unseres Interviews war TODOROV eher zurückhaltend, als wir ARON ins Spiel brachten; er zog es vor, auf Unterschiede hinzuweisen (,RAYMOND ARON war viel stärker auf das politische Tagesgeschehen fokussiert als ich“) oder über andere Denker - wie zum Beispiel Michail Bachtin, Louis Dumont und Germaine Tillion - zu sprechen, die ihn beeinflusst hatten. Ich konnte mich indessen nicht des Eindrucks erwehren, es handle sich bei dieser Abwehrhaltung um ein Paradebeispiel dessen, was Freud Reaktionbildung nennt, denn TODOROVS Antworten waren fast zu ausweichend. Tatsächlich liest sich sein 2003 verfasstes Vorwort zur Neuauflage von ARONS Memoiren wie ein Selbstporträt - das geschriebene Wort ist manchmal aufschlussreicher als die gesprochene Sprache (TODOROV 2003). Im nun folgenden, abschließenden Teil meiner Überlegungen möchte ich jenes Vorwort aus eben diesem Blickwinkel betrachten. Das erste, was dabei ins Auge springt, ist die scharfe, sowohl von TODOROV als auch von ARON unmissverständlich vorgebrachte Kritik an jeglicher Form des Totalitarismus. Es ist dies zugegebenermaßen kaum verwunderlich: ARON erlebte Anfang der 1930er Jahre in Köln und Berlin den Aufstieg des Nationalsozialismus, und TODOROV verbrachte seine Jugend im kommunistischen Bulgarien. Die Verwerfung solcher Pervertierungen des menschlichen Zusammenlebens scheint offensichtlich. (Ich beeile mich hinzuzufügen, dass diese kritische Position dennoch keineswegs von der gesamten französischen intellektuellen Welt geteilt wurde: Es gab nicht wenige penseurs, écrivains und artistes, die es schafften, die Augen vor der Realität des real-existierenden So- 
zialismus zu verschließen). ${ }^{5}$ Wesentlich schwieriger ist es, den Zukunftsverheißungen der totalitären Versuchung ${ }^{6} \mathrm{zu}$ widerstehen. Denn es gab und gibt auch und gerade in Frankreich Denker, die zwar die Schrecken des Totalitarismus als Schrecken anerkennen, aber trotzdem darauf bestehen, politischer Terror lasse sich im Namen eines zukünftigen Humanismus rechtfertigen - nach dem bereits von Pascal kritisierten Motto Nous corrigeons le vice du moyen par la pureté de la fin (Wir beheben die Lasterhaftigkeit des Mittels durch die Reinheit des Zwecks, PASCAL 1850:125). ${ }^{7}$ Im Gegensatz dazu haben TODOROV und ARON ,niemals versucht, das, was sich schlicht nicht verteidigen lässt, auf dialektischem Wege zu rechtfertigen“" (TODOROV 2003:XII). Noch auch haben sie jener anderen, ebenso perniziösen Form der totalitären Versuchung nachgegeben, jener, die uns glauben macht, es gäbe einen sicheren Weg ins irdische Paradies, einen Weg ohne Verbrechen und Blutvergießen. Im Gegensatz zu solchem Perfektionswahn erinnern uns TODOROV und ARON an die Pascalsche Evidenz, die wir immer wieder verdrängen: „der Mensch ist weder Engel noch Tier, und das Unglück will, dass wer Engel sein will, zum Tier wird“ (PASCAL 1897:82). ${ }^{8}$ Das Paradies liegt hinter uns; wir leben nicht mehr im Garten Eden, sondern in einem höchst unvollkommenen Garten, ${ }^{9}$ dessen einziger Ausgang zur Hölle führt.

Diese Entscheidung gegen den Totalitarismus ist zugleich eine Entscheidung für den modernen Rechts- und Sozialstaat, der einen Kompromiss zwischen Freiheit und Gleichheit, zwischen Individuum und Gesellschaft, zwischen Hoffnung und Resignation darstellt - einen Kompromiss, der „lediglich [...] weniger schlecht ist als die Alternativen“" (TODOROV 2003:XIX). Es ist diese Welt, in der TODOROV und ARON an sich entschieden haben zu leben und sich als Beobachter zu engagieren. Und in dieser dezidiert nicht-manichäischen Welt hat man es niemals mit dem Kampf des Guten gegen das Böse zu tun,

$5 \quad \mathrm{Zu}$ ARONS Kritik am Totalitarismus siehe insbesondere ARON 1957. Zum stalinistischen Engagement französischer Intellektueller siehe etwa die Standardwerke von: JUDT 1992 und LOTTMAN 1998. Den französisch-intellektuellen Flirt mit dem Maoismus analysiert WOLIN 2010.

6 [Anm. d. Übers.:] Zu diesem Begriff vgl. REVEL 1977.

7 Siehe etwa Merleau-Ponty 1990. Dass eine solche Position keineswegs der Vergangenheit angehört, lässt sich wohl am deutlichsten am Werk Slavoj Žižeks ablesen (vgl. dazu DE BERG 2015).

8 Das im Französischen sprichwörtlich gewordene Original lautet: 1'homme n'est ni ange ni bête, et le malheur veut que qui veut faire l'ange fait la bête. [Anm. d. Übers.:] Zu diesem Begriff vgl. ToDorov 1998. 
sondern immer nur mit Gradierungen, mit „,besser oder schlechter“ (TODOROV 2003:XIV). ${ }^{10}$ Dies ist der Grund, weshalb unsere Urteile niemals endgültig sein können; weshalb es immer Ungewissheiten geben wird; weshalb der Zweifel - der cartesianische, systematische Zweifel - etwas Positives ist; und warum die Arbeit der Erkenntnis immer weitergeht und immer weitergehen wird: „wie die Arbeit des Sisyphus“ (TODOROV 2003:XVIII). Dementsprechend haben TODOROV und ARON auch „keine Illusionen über die Wirksamkeit der Vernunft" (TODOROV 2003:XVIII). ${ }^{11}$ Sie sind sich sehr wohl bewusst, dass wissen und können zwei sehr verschiedene Dinge sind und dass wissen und wollen ebenfalls zweierlei sind: Nur zu oft bleiben unsere Einsicht und unsere Handlungen durch die Kluft menschlicher Unvollkommenheit getrennt. Vor diesem Hintergrund lässt sich TODOROVS abschließendes Lob ARONS als Widerspiegelung dessen verstehen, wonach er selber strebte:

Er entschied sich, ein Diener und Aufklärer der Öffentlichkeit zu sein, um so ein wenig Licht auf unsere Existenz zu werfen. Er erfüllte diese Aufgabe, indem er darauf verzichtete, den Traumverkäufer zu spielen, indem er jeden dazu ermutigte, die Welt zu erkennen, wie sie ist, und sie gerecht zu beurteilen. [...] Anstatt die Herzen zu entflammen, hat er versucht, den Verstand zu erleuchten; und er tat dies im Bewusstsein, dass das Licht, das er bringt, immer nur der flackernden Flamme einer Kerze gleicht, die ständig neu entzündet werden muss. (TODOROV 2003:XXVII) ${ }^{12}$

Dieser selbstreflexive Glaube an die Vernunft, ein Glaube, der nie in Absolutismus abrutscht, bildet den Kern des TODOROVschEN Denkansatzes. Heute, in einer Welt der zunehmenden Pseudosicherheiten des Ethno-Nationalismus und einer alteritätsversessenen Identitätspolitik ist dieses Plädoyer für die aufklärerischen Ideale der Toleranz, Gesprächsbereitschaft und Selbstkritik fast subversiv und auf alle Fälle wichtiger denn je.

Alle Übers. hieraus v. DAVID ENGELS.

TODOROV bemüht hier ein mündliches Zitat ARONS aus: BAVEREZ 1993:369.

[Anm. d. Übers.:] Ich habe diese Passage relativ frei übersetzt, um den Stil und die rhetorische Stoßrichtung des Textes zu erhalten. Das Original lautet: „Il a choisi d'être un serviteur et un éclaireur du public, un porteur de lumière. Il s'est acquitté de sa tâche en renonçant à jouer au marchand de rêves, en incitant chacun à connaître le monde qui l'entoure et à le juger en équité. [...] Plutôt que d'enflammer les cœurs, il a voulu éclairer les esprits; et encore, la lumière qu'il apporte ressemble, non à celle de l'éclair, mais à la flamme vacillante de la bougie qu'on rallume sans cesse“". 


\section{Literatur}

ARON, RAYMOND (1957): Opium für Intellektuelle oder die Sucht nach Weltanschauung. Aus dem Französischen von Klaus Peter Schulz. Köln. Erstausgabe 1955.

ARON, RAYMOND (1983): Der engagierte Beobachter. Gespräche mit Jean-Louis Missika und Dominique Wolton. Aus dem Französischen von Klaus Schomburg. Stuttgart. Erstausgabe 1981.

Baverez, Nicolas (1993): Raymond Aron. Paris.

DE BERG, HenK (2015): Fear of the Martians: On Slavoj Žižek's Uses of Argument. In: Paragraph 38.3:347-368.

DE Berg, Henk / ZBinden, Karine (2017): Le Bien est l'ennemi du mieux. Entretien avec Tzvetan Todorov. In: Books: L'actualité à la lumi ère des livres 83:12-14.

Finkielkraut, Alain (1989): Die Niederlage des Denkens. Aus dem Französischen von Nicola Volland. Reinbek bei Hamburg. Erstausgabe 1987.

Foucault, Michael (1977): Überwachen und Strafen. Die Geburt des Gefängnisses. Aus dem Französischen von Walter Seitter. Frankfurt a.M. Erstausgabe 1975.

Halimi, Serge (erweiterte, revidierte Fassung 2005): Les nouveaux chiens de garde. Paris. Erstausgabe 1997.

HAZAREesingh, SudHIR (2015): How the French Think. New York.

Hegel, Georg Wilhelm Friedrich (1986): Phänomenologie des Geistes. Frankfurt a.M. Erstausgabe 1807.

JUDT, TONY (1992): Past Imperfect: French Intellectuals, 1944-1956. Berkeley / Los Angeles.

Lottman, Herbert R. (1998): The Left Bank: Writers, Artists, and Politics from the Popular Front to the Cold War. Chicago.

Merleau-Ponty, Maurice (1990): Humanismus und Terror. Versuch über den Kommunismus. Aus dem Französischen von Eva Moldenhauer. Frankfurt a.M. Erstausgabe 1947.

Nizan, Paul (1969): Die Wachhunde. Essay. Aus dem Französischen von Traugott König. Reinbek bei Hamburg. Erstausgabe 1932.

N.N.: Tzvetan Todorov. In: Der Spiegel 7 v. 11. Februar 2017:123.

PASCAL, Blaise (1850): Les provinciales. Paris. Erstausgabe 1656-1657.

Pascal, Blaise (1897): Les Pensées. Paris. Erstausgabe 1670.

ReVEL, JEAn-FrançOIs (1977): Die totalitäre Versuchung. Aus dem Französischen von Eva Brückner-Pfaffenberger. Frankfurt a.M. Erstausgabe 1976.

TODOROv, TzVETAN (1998): Le jardin imparfait. La pensée humaniste en France. Paris.

Todorov, Tzvetan (2003): Préface. In: Aron, Raymond: Mémoires. Neuausgabe Paris. Erstausgabe 1983, VII-XXVII.

WiLlms, BERNARD (1969): Planungsideologie und revolutionäre Utopie. Die zweifache Flucht in die Zukunft. Stuttgart. 
WOLIN, RichARD (2010): The Wind from the East: French Intellectuals, the Cultural Revolution, and the Legacy of the 1960s. Princeton / Oxford.

ZEMMOUR, ERIC (2014): Le suicide français. Paris.

ŽIŽEK, SLAVOJ (2011): Living in the End Times. London. Erstausgabe 2010. 